\title{
Extreme soliton pulsations in dissipative systems
}

\author{
Wonkeun Chang, ${ }^{1}$ Jose M. Soto-Crespo, ${ }^{2}$ Peter Vouzas, ${ }^{1}$ and Nail Akhmediev ${ }^{1}$ \\ ${ }^{1}$ Optical Sciences Group, Research School of Physics and Engineering, The Australian National University, Acton ACT 2601, Australia \\ ${ }^{2}$ Instituto de Óptica, C.S.I.C., Serrano 121, 28006 Madrid, Spain
}

(Received 6 May 2015; published 26 August 2015)

\begin{abstract}
We have found a strongly pulsating regime of dissipative solitons in the laser model described by the complex cubic-quintic Ginzburg-Landau equation. The pulse energy within each period of pulsations may change more than two orders of magnitude. The soliton spectra in this regime also experience large variations. Period doubling phenomena and chaotic behaviors are observed in the boundaries of existence of these pulsating solutions.
\end{abstract}

DOI: 10.1103/PhysRevE.92.022926

\section{INTRODUCTION}

The concept of dissipative soliton is related to solitary waves in a medium in presence of gain and loss [1]. The concept can be equally well applied to either optical [2-4] or matter waves [5-8] as well as to the description of combined models when optical and matter waves are involved on equal basis [9]. One particular example of applicability of such a concept is the passively mode-locked laser [10]. The dissipative soliton concept is universal and can be applied to a large class of solid state lasers [11,12], semiconductor oscillators [13], fiber lasers [1418], and even to microresonators [19]. The usefulness of the concept is demonstrated with the development of modelocked fiber lasers with unprecedented output power levels [20,21].

One of the main techniques used in the theory of passively mode-locked lasers is the so called master equation approach [22]. This approach was first suggested by Haus [23,24], and later developed into a cubic-quintic Ginzburg-Landau equation (CGLE) as the equation of minimal complexity [25] that admits stable soliton solutions, thus allowing us to describe pulse generation phenomena by optical oscillators. The presence of several parameters in the CGLE makes the dynamical system highly complicated [26]. Each equation parameter can be related to actual parameters of a particular laser system [12] although the relation depends on the particular laser model and varies significantly from one model to another.

Although some solutions of the CGLE can be written in analytical form $[27,28]$, there is no way to give a complete description of all solutions. Various methods can be used to study isolated localized solutions. These are either numerical simulations or analytical approximations based on the reduction of a given infinite-dimensional dynamical system into a finite-dimensional one [29]. The latter methods allow us to find approximate solutions which still need to be confirmed with direct numerical simulations. In any case, the numerical integration remains the main tool in obtaining new solutions [30].

Depending on their propagation properties, localized solutions of the CGLE may belong to several major classes, namely, stationary, pulsating, and chaotic [26]. Each of them can be categorized into subclasses with more complicated evolution [31]. Stationary solitons are the easiest objects to model and to search using either approximate or numerical tools. Pulsating solitons are no less important than stationary
PACS number(s): 42.65.Sf, 42.60.Mi, 42.65.Tg

ones. In the parameter space, pulsating and stationary solitons usually exist in areas with common borders [26].

In contrast to solitons of Hamiltonian systems [32,33], dissipative solitons do exist whether the average dispersion in the cavity is normal or anomalous. This happens because of the composite balance required for dissipative solitons. This balance involves dispersion and nonlinearity and gain and loss rather than just dispersion and nonlinearity as for Hamiltonian solitons [33]. Consequently, dispersion can have any sign for the total balance to remain effective for a localized solution. Generally, anomalous dispersion provides easier conditions for this balance because it is matched with the balance achieved for the Hamiltonian solitons. Accordingly, the soliton temporal profile and spectrum are often close to sech functions. On the other hand, when dealing with normal dispersion cavities, we may expect more exotic solutions.

The master equation approach allows us to study complicated dynamics of passively mode-locked lasers within the frame of a single partial differential equation. This might seem simpler than operating with the complete set of laser equations. However, the analysis of the whole set of equation parameters where soliton solutions can be found is a tedious task. In this work, we present pulsating soliton solutions. The main feature of these solitons is their unusually high amplitude of pulsations, namely, the ratio of maximal energy of the pulse to the minimal energy within a period may exceed two orders of magnitude. Such large variations happen in the normal dispersion regime of the laser cavity. These kinds of pulsations were not observed in the past in the studies of dissipative solitons either in the anomalous [26] or normal dispersion regimes.

Most of the pulselike solutions of the CGLE found in numerical simulations have been observed experimentally in various passively mode-locked laser configurations. For example, the pulsating solutions predicted in [26] have been experimentally verified in a fiber ring laser [34], and a highly complex solution like the exploding soliton predicted in [31] has been observed experimentally in two completely different laser setups $[35,36]$. These two examples show that the master equation approach is a useful tool for predicting new phenomena in laser systems. As such, it guides and stimulates experimental work. The pulsating solutions that we have found in this work may and deserve to be observed experimentally.

\section{MODEL}

To be specific, we deal with the cubic-quintic complex Ginzburg-Landau equation (CGLE), which in its standard form 
reads as [10]

$$
\begin{aligned}
& i \psi_{z}+\frac{D}{2} \psi_{t t}+|\psi|^{2} \psi+v|\psi|^{4} \psi \\
& \quad=i \delta \psi+i \epsilon|\psi|^{2} \psi+i \beta \psi_{t t}+i \mu|\psi|^{4} \psi,
\end{aligned}
$$

where for passively mode-locked lasers, $t$ is the normalized time in a frame of reference moving with the group velocity, $\psi$ is the complex envelope of the optical field, and $z$ is the propagation distance along the unfolded cavity. The meaning of the equation parameters on the left-hand side is the following: $D$ denotes the cavity dispersion, being anomalous when $D>0$ and normal if $D<0$, and $v$ is the quintic refractive index coefficient. Dissipative terms are written on the right-hand side of (1), and the meaning of the corresponding parameters is the following: $\delta$ denotes linear gain and loss, $\beta$ is the gain bandwidth coefficient, and $\epsilon$ and $\mu$ are the cubic and quintic gain and loss coefficients, respectively.

Several types of periodic solutions to this equation with unusual behaviors have been obtained in the past in the anomalous dispersion regime [31]. Contrarily to the present case, those solutions experienced periodic changes mostly in their width, while keeping almost constant their peak amplitude. Here, it is the peak amplitude that largely changes periodically rather than the width. We consider these amplitude variations to be extreme. In fact, this specific type of evolution requires a special care in numerical procedures.

To make sure that the numerical results we are presenting here are correct, we used two different programs and three different computing platforms. We obtained identical results in all cases. Namely, we used a split-step technique with a fourth-order Runge-Kutta algorithm for solving the nonlinear part of the equation, while the linear part was solved in Fourier space. Modifications of this technique [37] have also been used. We used step sizes, small enough to be sure that the parts of the solution with sharply growing amplitudes are finely discretized along both $z$ and $t$ axes. Namely, for the most extreme cases we used up to 524288 mesh points in transverse direction to sample the $t$ interval up to $[-200,200]$, that is sufficiently large to have practically zero values of the envelope function at its edges. With this choice, the spectrum of the localized solutions is also well sampled in Fourier domain. In general, it was sufficient to use 16384 points to scan a 10 times smaller temporal interval. In the longitudinal direction we used a step length as small as $10^{-6}$, although the use of an adaptive step-size technique relieved the requirements and allowed us to use bigger step sizes.

\section{PULSATING SOLITONS}

Figure 1(a) shows a typical example of the solutions we have found numerically for the set of parameters given in the caption. It shows pulsations of a single pulse. The unusual feature of these pulsations is that they lead to a recurrent sharp growth of the amplitude of the pulse followed by an even sharper drop. The solution can be considered as a periodic sequence of spikes. The duration of the extra-high amplitude spikes is significantly shorter than the period of pulsations. In this example, the pulse amplitude increases five times following its drop quickly to its original value. Moreover, the


FIG. 1. (Color online) (a) Periodic emergence of spikes by the pulsating soliton found for the following set of parameters of Eq. (1): $D=-1.0, v=0.1, \delta=-0.1, \beta=0.125, \epsilon=0.95$, and $\mu=-0.001$. (b) The evolution of the spectrum for the same simulation. The spectrum expands approximately eight times when the pulse reaches its highest amplitude. Another specific feature of the spectrum is the set of sidebands at fixed frequencies generated after the spike.

spike creates two side pulses that diverge and extinguish soon on further propagation. The spectrum evolution of the pulse is shown in Fig. 1(b). The spectrum naturally widens at the position of the spike creating subsequently discrete sidebands symmetrically located at each side. The latter gradually decay before the next spike is generated.

To illustrate further the pulse evolution, Fig. 2 shows one period of oscillations in a three-dimensional (3D) format.

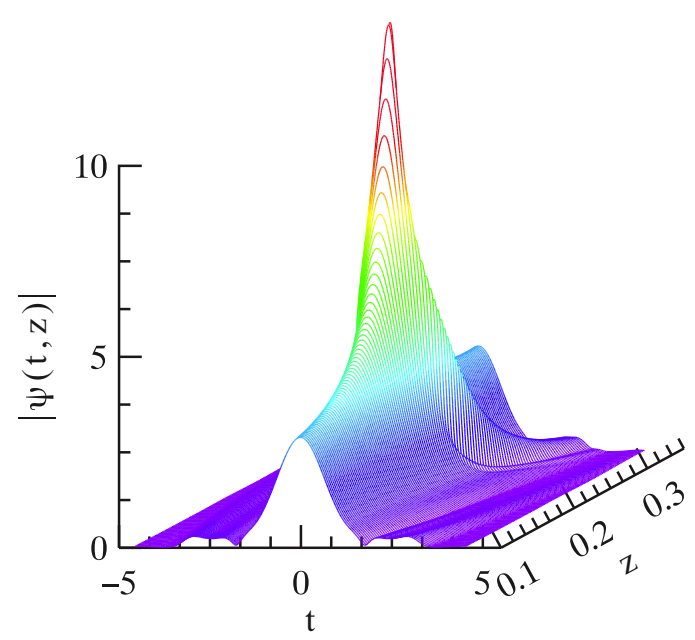

FIG. 2. (Color online) Pulse profile evolution of the same solution as in Fig. 1 during one period. The pulse experiences a sharp amplitude increase while keeping its width nearly constant. The spike then decays and two smaller pulses leave the central one symmetrically on each side. They gradually vanish. This can be seen more clearly in Fig. 1(a). 




FIG. 3. Pulse peak amplitude evolution for the same solution as in Fig. 1. The two vertical dotted lines are the boundaries of the $z$ interval of evolution in Fig. 2 . The highest amplitude $\approx 12.5$ exceeds approximately five times the lowest amplitude that is $\approx 2.7$.

The spike grows from a background pulse that has to be of certain minimal amplitude. If the amplitude drops below a critical value, the pulse will experience loss and will decay to zero. Explanations for this can be found in [38]. The two characteristic continuous wave solutions $(\mathrm{CW})$ for the chosen set of parameters have amplitudes $A_{1}=0.3245$ and $A_{2}=30.82$, respectively. These numbers provide approximate values for the potential peak amplitudes (see Figs. 2 and 3 of Ref. [38]).

In our case, the minimal peak amplitude of the soliton exceeds slightly 2 , and it is well above $A_{1}$. The pulses with amplitude smaller than $A_{1}$ decay because the coefficient $\delta$ in front of the linear gain and loss term is negative. Consequently, all solutions below the critical value that we estimate to be around 0.3245 will disappear. The limit is actually slightly higher because of the presence of the spectral loss term: the coefficient $\beta$ being positive, increases this limit. To give an example of such dissipation, the two side pulses around the main soliton, with amplitudes smaller than the critical value, decay as it can be seen from Figs. 1 and 2.

Figure 3 shows the evolution of the peak amplitude of the soliton at its center, $t=0$. This figure shows once again the quick increase of the pulse amplitude and its subsequent quick decay. Pulsating solitons are not unusual for the CGLE as we know from previous works [26,31]. However, the ratio of the maximal amplitude to the minimal one in these works was far from the values shown in this example. Moreover, the ratio here is still far from the maximal one that we can obtain for other values of the parameters, as we will see in the following.

The main characteristic of pulses measured in the optics experiments is their energy. We have calculated the total pulse energy $Q(z)$ according to

$$
Q(z)=\int_{-\infty}^{\infty}|\psi(z, t)|^{2} d t
$$

The evolution of $Q$ along $z$ is shown in Fig. 4. The variation of energy in each period of oscillations is even higher than for the amplitude. The peak energy in each period exceeds 15 times the minimal energy. Comparing the location of peaks in Figs. 3 and 4 , we can see that the growth of energy is related to the growth of the central part of the solution. However, the exact maxima of the peak amplitude are slightly delayed relative to the maxima of the energy.

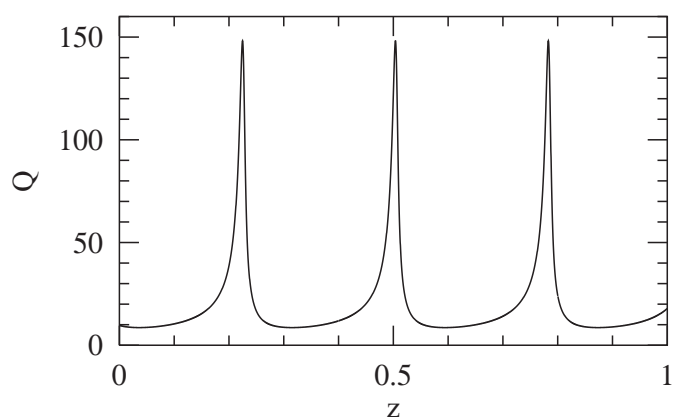

FIG. 4. Pulse energy evolution for the same solution as in Fig. 1.

The strong variations of the pulse parameters are far from being a general feature of the pulsating solitons. Variations are found to be stronger in the normal dispersion regime, i.e., when $D$ is negative. Figure 5 shows the variation of the maximal and minimal values of energy when the dispersion parameter $D$ is changed in the interval $[-1,0]$. The dashed red line shows the maxima of energy $Q_{M}$, while the dotted blue line represents the minima $Q_{m}$. The presence of two separate curves for $Q_{M}$ and $Q_{m}$ is an indication that we are dealing with pulsating solutions. The solid green curve in Fig. 5 shows the ratio of $Q_{M}$ to $Q_{m}$. Although the maximal energy mostly decreases when we move towards $D \approx-0.5$, the ratio of maximal energy to the minimal one steadily increases. The absolute maximum of this ratio occurs at the point $D \approx-0.5$ as the green solid curve shows. According to this plot, the ratio of $Q_{M}$ to $Q_{m}$ may reach values of up to 120 . There are four segments of $D$ where we have found pulsating solitons. The right-hand side interval continues into the region of positive $D$. The ratio $Q_{M}$ to $Q_{m}$ is smaller in other intervals of pulsating soliton existence. The three gray areas of this plot indicate the values of $D$ for which any localized solution disappears on propagation.

Figure 5 does not have sufficient resolution to show the fine structure of the diagram near the borders with the gray areas. In order to see this fine structure, we plot, in

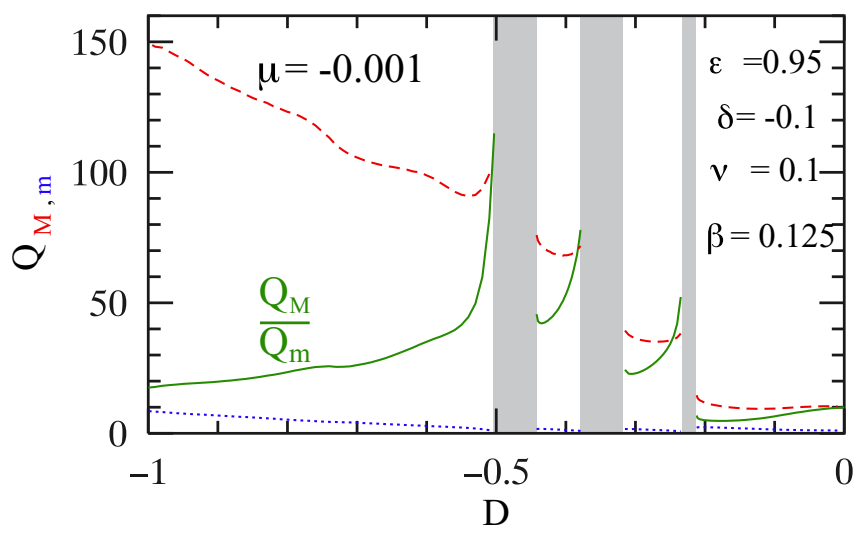

FIG. 5. (Color online) Bifurcation diagram representing the maximal $Q_{M}$ (red dashed line) and minimal $Q_{m}$ (blue dotted line) values of the energy of the periodic solution vs $D$. Other parameters, written in this figure, are the same as in Fig. 1. The ratio $Q_{M} / Q_{m}$ versus $D$ is shown by the solid green curve on the same dimensionless scale as $Q$. In the gray vertical areas, solutions vanish on propagation, no matter what kind of initial conditions are used. 


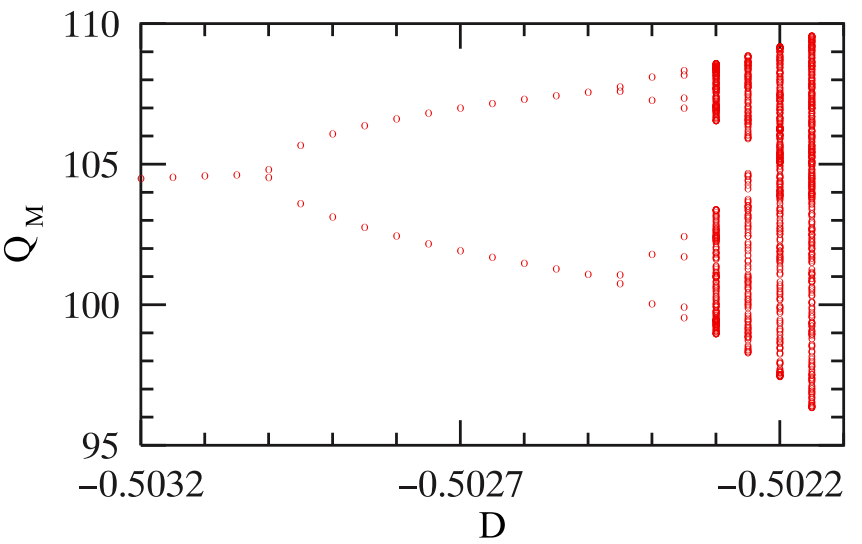

FIG. 6. (Color online) Magnification of a small part of the bifurcation diagram of Fig. 5 near the first gray stripe. Only maxima of the energy $Q_{M}$ are plotted in this diagram. Period doubling bifurcation of solitons occurs at $D \approx-0.503$. It follows by period quadrupling at $D \approx-0.50245$. Chaotic evolution can be observed in a narrow region of $D$ around $\approx-0.5022$.

Fig. 6, the maximal values of the energy with a much smaller separation of $D$ values than in Fig. 5. This higher resolution plot shows that the maximal energy $Q_{M}$ splits into two branches at $D \approx-0.50295$. The maximum of energy takes one of the two alternative values every second period. This switch corresponds to a period doubling bifurcation for pulsating solitons. The two branches of energy split into four at $D \approx-0.50245$. This is a transition into the region of period quadrupling of pulsating solitons. Although we cannot resolve the whole sequence of period doubling bifurcations, we observe that the pulse evolution enters the chaotic regime in the narrow region around $\approx-0.5022$. This is seen in this figure in the form of two continuous vertical lines of dots. Each dot is a realization of random value of maximal energy in consecutive periods of pulsations. The dots fill continuously the two finite intervals along the two vertical lines at the right-hand side of the plot.

We studied the pulse evolution more carefully at the point $D=-0.5032$ located at the left-hand side of the first period doubling bifurcation. The pulse center amplitude evolution at this point is shown in Fig. 7. It is similar to the previous diagram in Fig. 3. However, the ratio of maximal amplitude to the minimal one here is much higher. It is close to 20 . The ratio of the period to the duration of the spike is also much higher. For the sets of parameters that we studied here, this is one of the highest ratios that we obtained within the interval $[-1<D<0]$.

The periodic energy evolution for this case is shown in Fig. 8. The ratio of highest energy to the minimal one is also significantly larger here. It reaches the value of 115 . This is much higher than at $D=-1$. Moreover, the peak energy $Q_{M}$ is much higher than the energy averaged over $z$. The latter is shown by the dashed blue line in Fig. 8 above the gray area. This is an interesting result by itself. In terms of laser applications, it means that the pulse periodically can reach exceptionally high energies leaving the average energy inside the laser cavity at relatively low values. This would be beneficial for generating record high energy output pulses

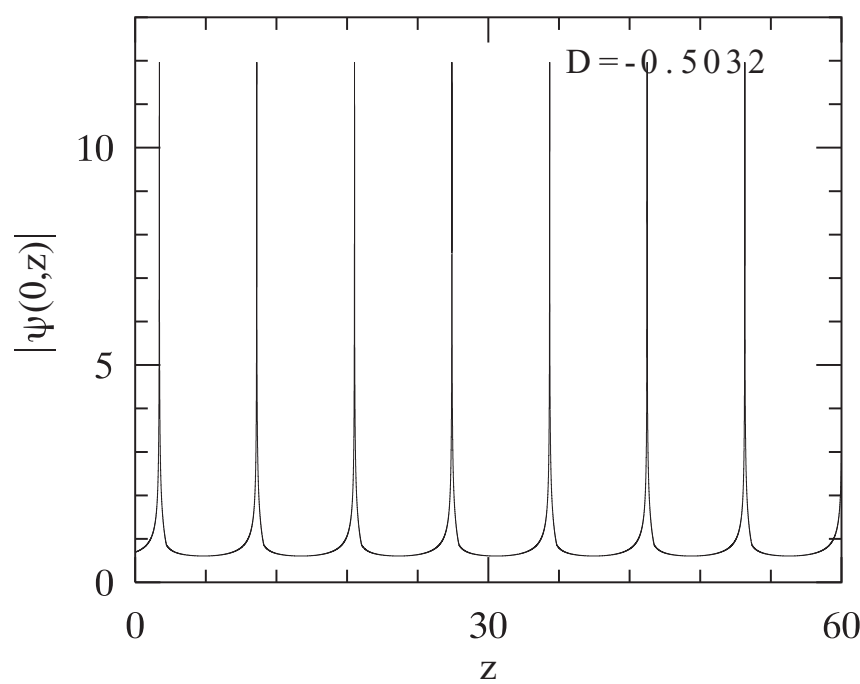

FIG. 7. Central amplitude evolution of the soliton at the point $D=-0.5032$ of the bifurcation diagram of Fig. 6 , i.e., to the left of the point of period doubling bifurcation. This is an example of the most extreme oscillations: the maximum of the peak amplitude exceeds almost 20 times the minimum amplitude. The period is also larger than in the case of Fig. 3 while the spatial width of the spike is significantly smaller than the period.

while keeping the average powers within the equipment below dangerously high values.

Figure 9(a) shows the pulse amplitude profiles at the points of maximal and minimal amplitudes. We can see that the pulse width remains almost the same while the pulse amplitude varies more than an order of magnitude within the period. Figure 9(b) shows the pulse spectrum at these two points. The changes of the spectrum are especially impressive. Both the width and the height of the spectrum increase many times. At the upper extreme point, most part of the spectral energy

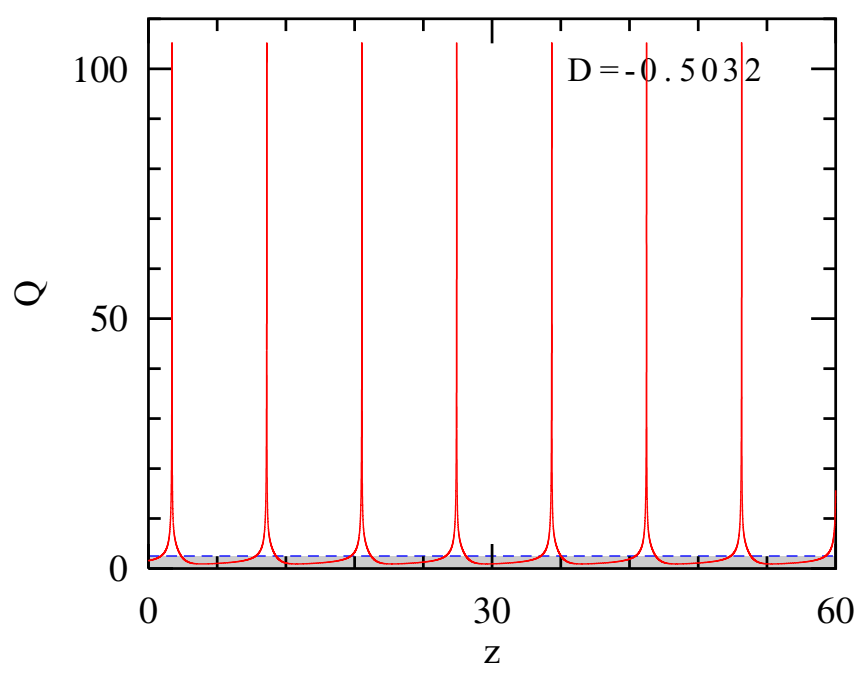

FIG. 8. (Color online) The energy evolution of the soliton for the same data as in Fig. 7. This is an example of the most extreme pulsations: the maximum energy exceeds the minimum energy more than 115 times. The average energy is shown by the blue dashed line above the gray area in this plot. 

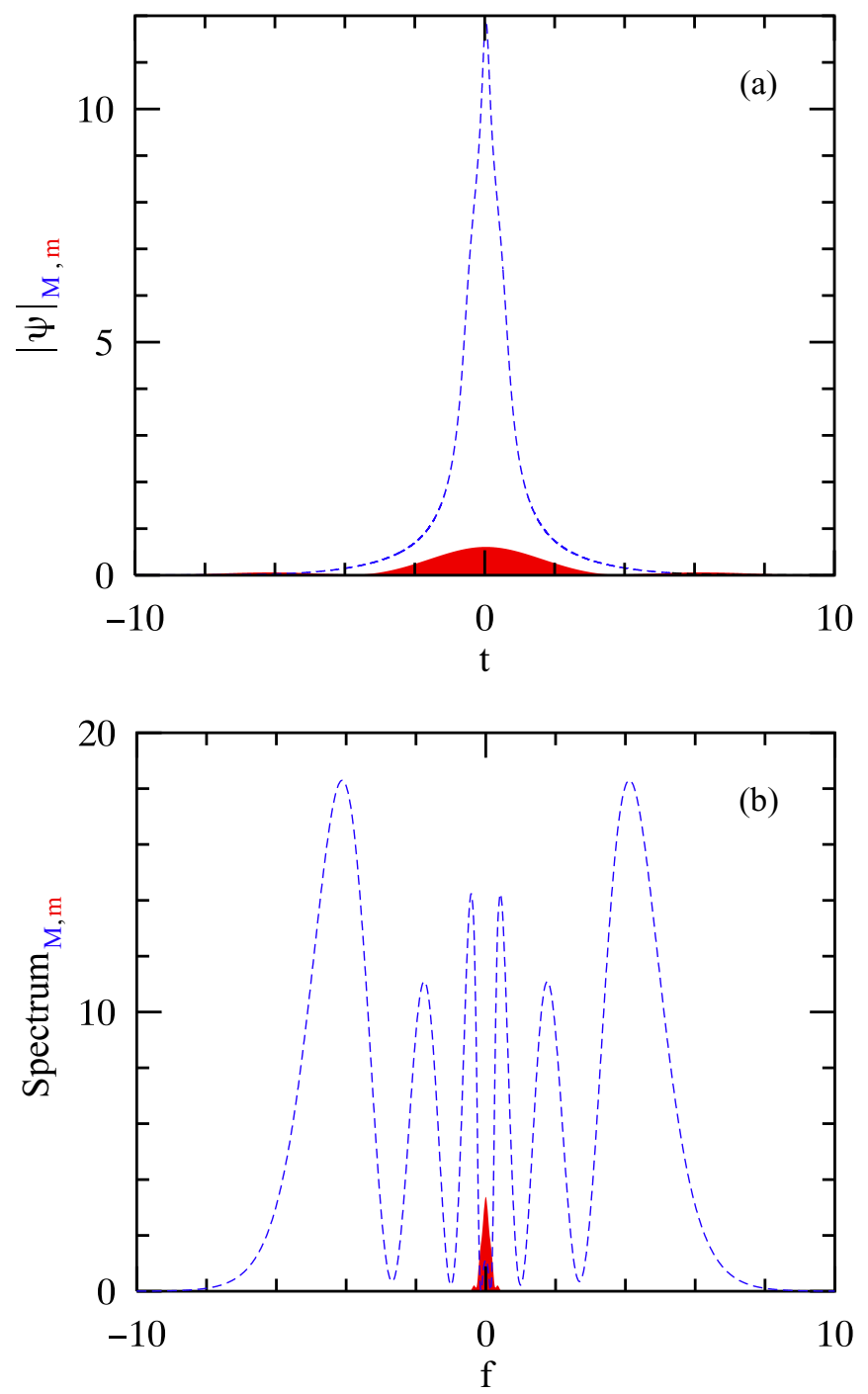

FIG. 9. (Color online) Extreme changes of (a) the shape and (b) the spectrum that the pulse experiences in a single period for $D=$ -0.5302 . The two profiles in (a) are shown for the pulse when it has the maximum amplitude (blue dashed curve) and minimal amplitude (red area). The two spectra in (b) correspond to the same profiles. The spectrum of the pulse widens significantly and splits into several sidebands with each one stronger than the spectrum shown in red.

is contained within several side lobes of the spectrum. Each side lobe is larger than the original spectrum at the point of minimum shown in red. Side lobe positions have roughly fixed frequencies during evolution as it can be seen from Fig. 1(b). This shows that the four-wave mixing is playing the main role in the formation of the spectrum. The phenomenon of spectrum widening can be beneficial in laser applications where the generation of the widest possible spectrum is required.

We have also constructed bifurcation diagrams for some other values of $\mu$, keeping the rest of the parameters fixed. Figure 10 shows the corresponding bifurcation diagram for $\mu=-0.003$. In this case, in addition to pulsating solitons, we obtained stationary solitons with fixed profile and therefore fixed amplitude. The black solid line in this diagram shows the amplitude of the stationary solitons. These solutions become



FIG. 10. (Color online) Bifurcation diagram vs $D$ when parameter $\mu=-0.003$. The solid black line represents the energy $Q$ of stationary solutions while the red dashed line (blue dotted line) represents the maxima (minima) of the energy of pulsating solitons.

periodic at the bifurcation point $D \approx-0.63$. To the right of this point, the dashed red line represents the maximum energy $Q_{M}$ of periodic solutions while the dotted blue line shows their minimum energy $Q_{m}$. We have not observed any period doubling for these parameters. The energy oscillations are also smaller here in comparison with the previous case. However, they increase when we increase $\mu$ (decrease $|\mu|)$.

Figure 11 shows a bifurcation diagram for the same set of parameters as before but now we have taken $\mu$ to be $\mu=$ -0.002 . The energy oscillations now occur from $Q_{m}=8$ to $Q_{M}=60$ at $D=-0.8$. As in the previous case, there are no period multiplications here. However, the diagram shows a discontinuity around $D=-0.227$. In the process of increasing the absolute value of $D$, starting from $D=0$, the solution

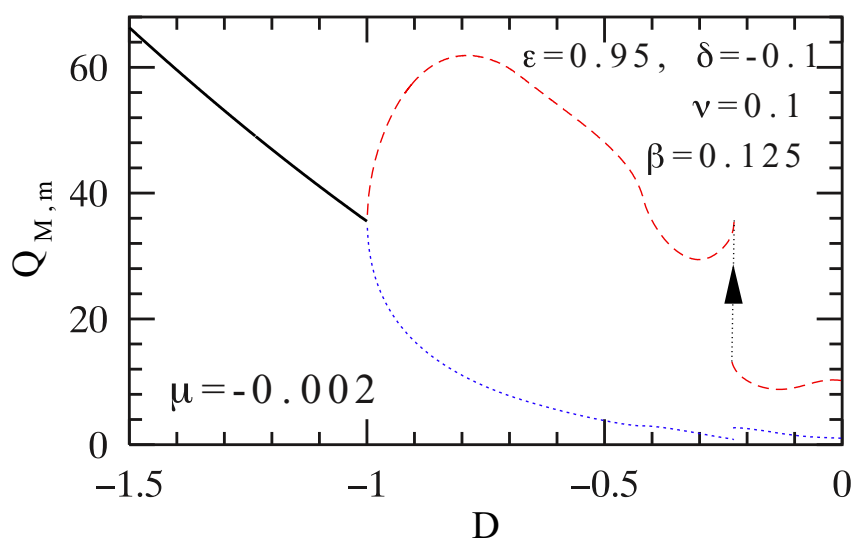

FIG. 11. (Color online) Bifurcation diagram vs $D$. Parameter $\mu=-0.002$. The solid black line represents the energy $Q$ of stationary solutions while the red dashed line (blue dotted line) represents the maxima (minima) of energy of the pulsating solitons. This diagram has a discontinuity: when moving from the left (increasing $D$ ) the solution vanishes at $D=-0.226$. Another branch of pulsating solutions with smaller amplitudes starts at slightly larger $D$. When reaching the discontinuity point from the right, the solution jumps directly to the left-hand side branch. This transition is indicated by the black arrow. 


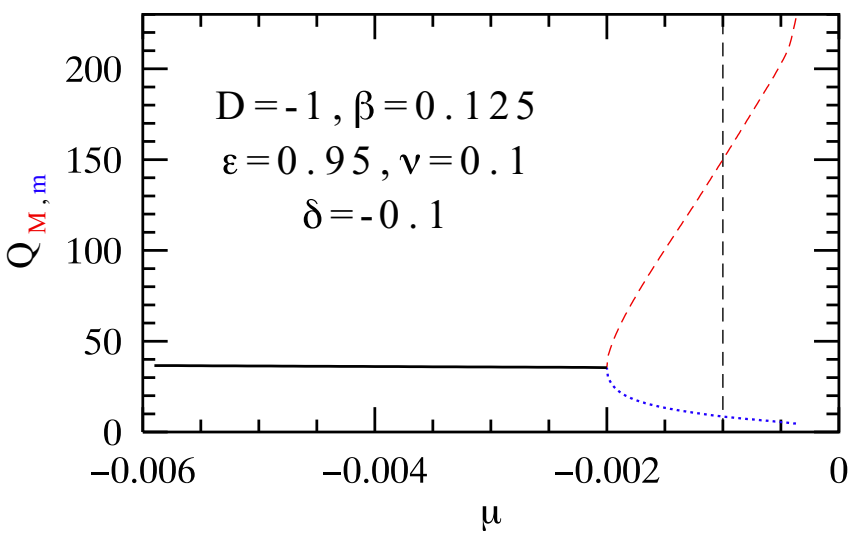

FIG. 12. (Color online) Bifurcation diagram vs $\mu$. The soliton is stationary with fixed energy $Q$ on the left-hand side of $\mu \approx-0.002$. The soliton becomes periodic to the right-hand side of this value. The minimal $Q_{m}$ (dotted blue curve) and maximal $Q_{M}$ (dashed red curve) values of $Q$ quickly diverge with the increase of $\mu$. The dashed black vertical line corresponds to the example in Fig. 1. The upper limit of $Q_{M}$ at $\mu \approx-0.0004$ seems to be unlimited.

jumps from one branch of periodic solitons to another one at $D \approx-0.22$. Moving in the opposite direction in $D$, the periodic solution vanishes when reaching the same point.

In order to see the $\mu$ dependence in more detail, we fixed $D=-1.0$ and varied $\mu$ in the interval from -0.006 to -0.0004 . The bifurcation diagram along the $\mu$ axis is shown in Fig. 12. The solid black horizontal line here corresponds to stationary solitons. The energy of these solitons


FIG. 13. (Color online) Evolution of (a) the profile, (b) the spectrum, and (c) the energy of the pulsating soliton very close to the bifurcation point $\mu=-0.002$ in Fig. 12. Energy in this case oscillates almost harmonically.
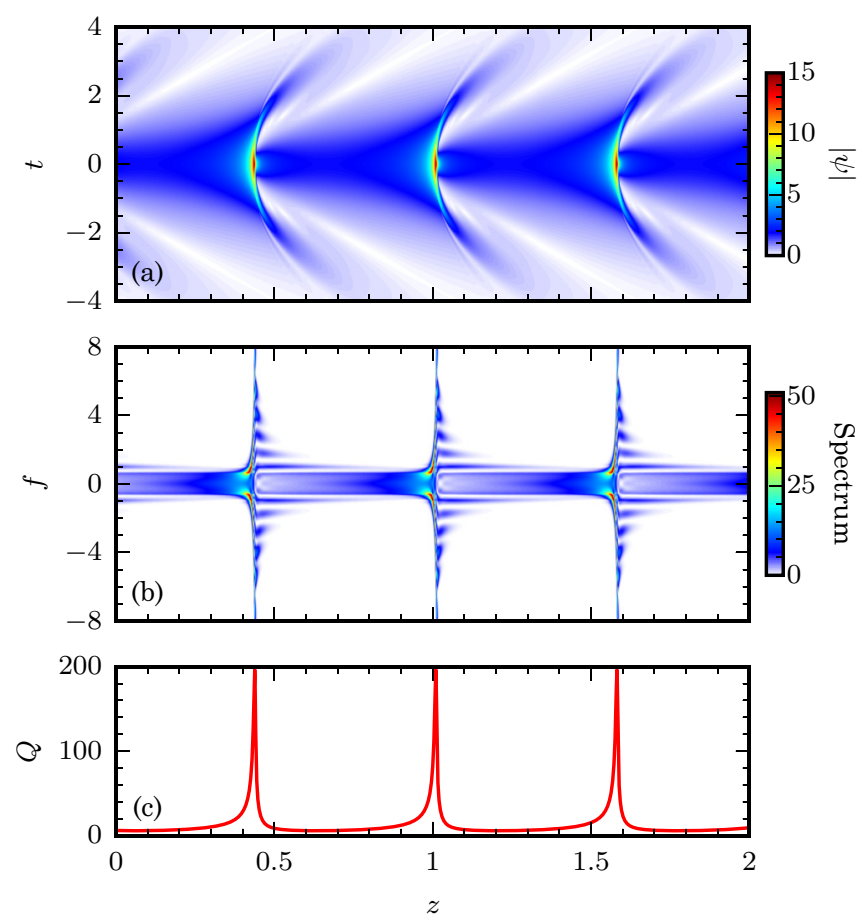

FIG. 14. (Color online) Evolution of (a) the profile, (b) the spectrum, and (c) the energy of the pulsating soliton at the point $\mu=-0.0005$ of the bifurcation diagram in Fig. 12. Pulsations are highly nonlinear.

is approximately constant $Q=38$ although the profile changes with $\mu$. At $\mu=-0.002$, stationary solitons are transformed into pulsating ones with quickly diverging values of minimal and maximal energies.

An example of pulsating soliton at $\mu=-0.002$ slightly above the bifurcation point is shown in Fig. 13. The oscillations

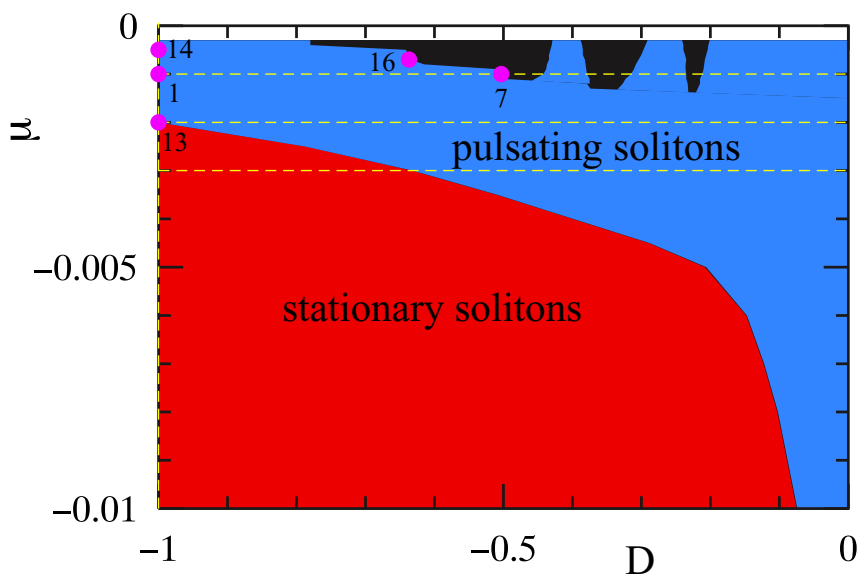

FIG. 15. (Color online) Areas of existence of (red) stationary and (blue) pulsating solitons on the $(D, \mu)$ plane. Solutions disappear in the black spots. The thick pink dots correspond to the solutions given in the figures above labeled with the same number. The vertical and horizontal dashed lines are chosen to construct the bifurcation diagrams shown above. The solutions cannot be resolved numerically for values of $\mu$ that are too small as in the white stripe on top of this plot. 
are small and the soliton profile and spectrum are changed only by a small amount. The changes of energy are also small and oscillations are almost harmonic. This can be seen in Fig. 13(c). However, when increasing $\mu$, the soliton oscillations become larger. This is shown in Fig. 14. The energy here changes in a much larger interval, namely, from 5 to almost 200. The whole dynamics now resembles the one in Fig. 1 although the energy oscillations are much higher here. Moreover, the amplitude of the energy oscillations did not reach its highest possible value as we can see from the bifurcation diagram Fig. 12. In principle, the energy difference in a period can reach even higher values.

A broader picture of soliton transformations could be seen from two-dimensional existence diagrams. The areas of existence of stationary and pulsating solitons on the $(D, \mu)$ plane are shown in Fig. 15. Bifurcation diagrams above are obtained on a vertical or horizontal straight line across this plot. These lines are marked with dashed yellow lines in this figure. The location of the parameters chosen for the particular
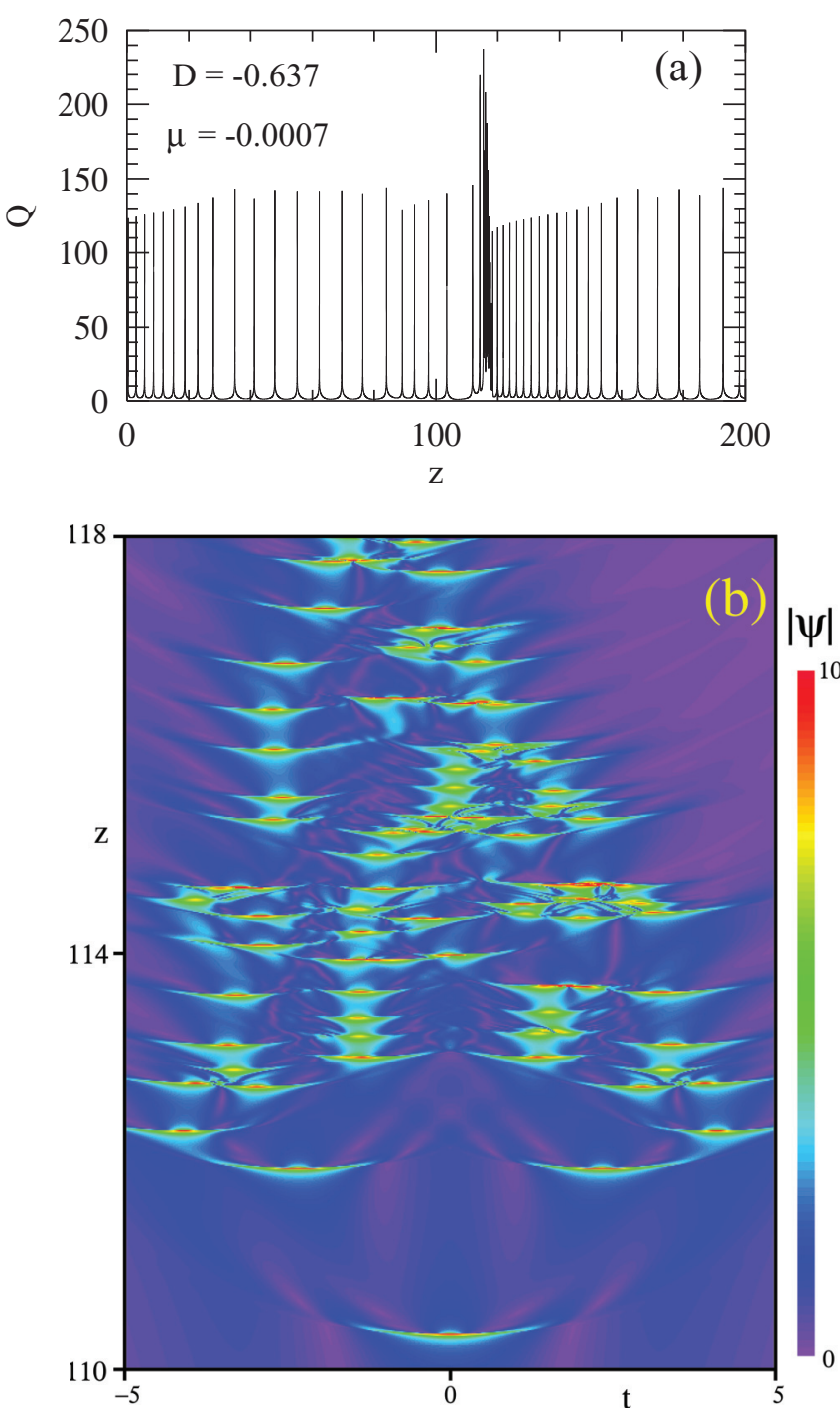

FIG. 16. (Color online) (a) Soliton energy evolution in the area of chaotic dynamics with $\mu=-0.0007$ and $D=-0.637$. The false color plot in (b) shows the soliton splitting and merging at around the point $z \approx 110$ in (a). solutions presented above are marked by thick pink dots. The numbers next to these dots correspond to the numbers of the figures where they are shown. The smallest possible values of $\mu$ when numerical simulations still provide reliable results are bounded by the white horizontal stripe in Fig. 15. For $\mu$ inside this white area, the soliton amplitude becomes too high and simulations may not describe stable solutions. Black spots in this diagram are the areas where solutions vanish on propagation. The boundaries around these spots are the regions where soliton solutions may become chaotic. An example of such transformation is shown in Fig. 6 above.

Another interesting example of chaotic soliton evolution is shown in Fig. 16. The evolution is mostly periodic but the period is not well defined and changes from one burst of energy to another. The periodicity is completely wrecked at around $z \approx 110$. The reason for this is the splitting of the soliton into two, followed by its division in a number of smaller pulses that subsequently merge into one. There is only one such event in the interval of $z$ from 0 to 200. However, this happens many times during the evolution for longer propagation distances.

\section{XFROG DIAGRAM}

A powerful method of short pulse investigations is the cross-correlation frequency-resolved optical gating (XFROG) technique developed in [39]. In order to facilitate the comparison of our results with potential experiments, we calculated the XFROG diagram for the pulses in Fig. 1. One such diagram is shown in Fig. 17. It is calculated for the pulse when the spike reaches the maximum amplitude. The spectrum in this case is close to be the widest and similar to the example shown in Fig. 9(b). An interesting detail in this XFROG diagram is that all side lobes at the negative and positive frequencies of the spectrum are related to the left-hand and right-hand side trails of the pulse, respectively.

The complete progressing of the XFROG diagram during the pulse evolution can be seen in the video attached as Supplemental Material [40]. The video shows the sharp expansion of the spectrum when the pulse amplitude takes

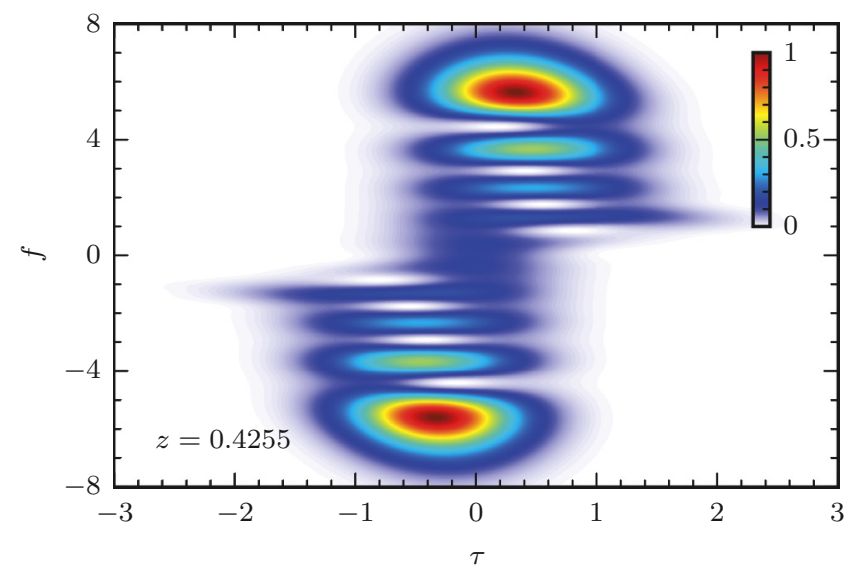

FIG. 17. (Color online) The XFROG diagram of the pulse when its maximal amplitude is about to be reached. The spectrum of the pulse widens significantly and splits into several highly energetic sidebands. The sidebands on each side are specifically related to one of the trails of the pulse in the time domain. 
extremely high values. This effect can be potentially used for pulse generation with extra-wide spectra directly by the laser oscillator without the need of special "supercontinuum generating" fibers.

\section{CONCLUSIONS}

In conclusion, based on the master equation approach, we have found some regimes of operation where laser oscillators may generate pulsating solitons with extreme ratios of maximal to minimal energies in each period of pulsation. This may happen when the laser has average normal cavity dispersion. We have found the parameters of the master equation that allow this to happen. Within the range of parameters considered in our work, we have found a particular case when the maximal energy in the period may exceed the minimal energy by more than two orders of magnitude. The highly energetic spikes repeatedly emitted by the soliton are much narrower than the period of pulsations. The highest spike energy is significantly larger than the average energy of the soliton. This phenomenon could be useful in choosing the regimes of laser operation with extreme bursts but small average powers within the cavity.

\section{ACKNOWLEDGMENTS}

The authors acknowledge the support of the Australian Research Council (Grants No. DE130101432, No. DP140100265, and No. DP150102057). The work of J.M.S.-C. was supported by MINECO under Contract No. TEC2012-37958-C02-02, and by Comunidad Autonoma de Madrid under Contract No. S2013/MIT-2790. J.M.S.-C. and N.A. acknowledge the support of the Volkswagen Foundation.
[1] Dissipative Solitons, edited by N. Akhmediev and A. Ankiewicz, (Springer, Berlin, 2005).

[2] D. Turaev, A. G. Vladimirov, and S. Zelik, Phys. Rev. Lett. 108, 263906 (2012).

[3] D. Mihalache, P. Romanian Acad. A 11, 142 (2010).

[4] S. Chen, Y. Liu, and A. Mysyrowicz, Phys. Rev. A 81, 061806(R) (2010).

[5] M. Argentina, P. Coullet, and V. Krinsky, J. Theor. Biol. 205, 47 (2000).

[6] C. Cartes, O. Descalzi, and H. R. Brand, Eur. Phys. J. Spec. Top. 223, 2145 (2014).

[7] H.-G. Purwins, H. U. Bödeker, and Sh. Amiranashvili, Adv. Phys. 59, 485 (2010).

[8] A. W. Liehr, Dissipative Solitons in Reaction Diffusion Systems (Springer, Berlin, 2013).

[9] N. Akhmediev, J. M. Soto-Crespo, and H. R. Brand, Phys. Lett. A 377, 968 (2013).

[10] Ph. Grelu and N. Akhmediev, Nat. Photonics 6, 84 (2012).

[11] V. L. Kalashnikov, E. Podivilov, A. Chernykh, S. Naumov, A. Fernandez, R. Graf, and A. Apolonski, New J. Phys. 7, 217 (2005).

[12] A. I. Korytin, A. Yu. Kryachko, and A. M. Sergeev, Radiophys. Quantum Electron. 44, 428 (2001).

[13] E. A. Ultanir, G. I. Stegeman, D. Michaelis, C. H. Lange, and F. Lederer, Phys. Rev. Lett. 90, 253903 (2003).

[14] A. E. Bednyakova, S. A. Babin, D. S. Kharenko, E. V. Podivilov, M. P. Fedoruk, V. L. Kalashnikov, and A. Apolonski, Opt. Express 21, 20556 (2013).

[15] W. H. Renninger, A. Chong, and F. W. Wise, Phys. Rev. A 77, 023814 (2008).

[16] F. Amrani, A. Haboucha, M. Salhi, H. Leblond, A. Komarov, and F. Sanchez, Appl. Phys. B 99, 107 (2010).

[17] D. Mortag, D. Wandt, U. Morgner, D. Kracht, and J. Neumann, Opt. Express 19, 546 (2011).

[18] R. Wang, Y. Dai, L. Yan, J. Wu, K. Xu, Y. Li, and J. Lin, Opt. Express 20, 6406 (2012).

[19] T. Herr, V. Brasch, J. D. Jost, C. Y. Wang, N. M. Kondratiev, M. L. Gorodetsky, and T. J. Kippenberg, Nat. Photonics 8, 145 (2013).
[20] K. Kieu, W. H. Renninger, A. Chong, and F. W. Wise, Opt. Lett. 34, 593 (2009).

[21] B. Chichkov, K. Hausmann, D. Wandt, U. Morgner, J. Neumann, and D. Kracht, Opt. Lett. 35, 2807 (2010).

[22] F. X. Kärtner, Few-Cycle Laser Pulse Generation and Its Applications (Springer, Berlin, 2004).

[23] H. A. Haus, J. Appl. Phys. 46, 3049 (1975).

[24] H. A. Haus, IEEE J. Sel. Top. Quantum Electron. 6, 1173 (2000).

[25] J. D. Moores, Opt. Commun. 96, 65 (1993).

[26] N. Akhmediev, J. M. Soto-Crespo, and G. Town, Phys. Rev. E 63, 056602 (2001).

[27] N. M. Akhmediev, V. V. Afanasjev, and J. M. Soto-Crespo, Phys. Rev. E 53, 1190 (1996).

[28] D. S. Kharenko, O. V. Shtyrina, I. A. Yarutkina, E. V. Podivilov, M. P. Fedoruk, and S. A. Babin, J. Opt. Soc. Am. B 28, 2314 (2011).

[29] E. N. Tsoy, A. Ankiewicz, and N. Akhmediev, Phys. Rev. E 73, 036621 (2006).

[30] V. L. Kalashnikov and E. Sorokin, Opt. Express 22, 30118 (2014).

[31] J. M. Soto-Crespo, N. Akhmediev, and A. Ankiewicz, Phys. Rev. Lett. 85, 2937 (2000).

[32] L. F. Mollenauer and G. P. Gordon, Solitons in Optical Fibers (Elsevier, London, 2006).

[33] N. Akhmediev and A. Ankiewicz, in Optical Solitons: Theoretical and Experimental Challenges, edited by K. Porsezian and V. C. Kurakose (Springer, Berlin, 2003), p 105.

[34] J. M. Soto-Crespo, M. Grapinet, Ph. Grelu, and N. Akhmediev, Phys. Rev. E 70, 066612 (2004).

[35] S..T. Cundiff, J. M. Soto-Crespo, and N. Akhmediev, Phys. Rev. Lett. 88, 073903 (2002).

[36] A. F. J. Runge, N. G. R. Broderick, and M. Erkintalo, Optica 2, 36 (2015).

[37] J. Hult, J. Lightwave Technol. 25, 3770 (2007).

[38] J. M. Soto-Crespo, N. Akhmediev, and G. Town, J. Opt. Soc. Am. B 19, 234 (2002).

[39] S. Linden, H. Giessen, and J. Kuhl, Phys. Status Solidi B 206, 119 (1998).

[40] See Supplemental Material at http://link.aps.org/supplemental/ 10.1103/PhysRevE.92.022926 for video of XFROG diagram evolution of the pulsating soliton. 